\title{
DNA ligase I is dephosphorylated during the execution step of etoposide-induced apoptosis
}

\section{Dear Editor,}

The activity of many factors involved in the control of DNA replication is regulated by phosphorylation/dephosphorylation events. ${ }^{1}$ In this respect, we have previously investigated the modulation of human DNA ligase I (hLigl), which is the activity involved in joining of Okazaki fragments during lagging strand synthesis. ${ }^{2}$ According to its function, the enzyme is a constituent of the replication factories that assemble during S-phase ${ }^{3}$ and plays also a role in DNA repair. ${ }^{4,5}$ We have previously shown that the recruitment of hLigl to replication factories is mediated by a short sequence referred as RFTS (Replication Factory Targeting Sequence) that corresponds to an evolutionary conserved PCNA binding site. ${ }^{6}$ The interaction with PCNA affects also the phosphorylation status of Ser66 of hLigl during the cell cycle. ${ }^{7}$ More recently, we have observed that in HeLa cells etoposide-induced double strand breaks (DSBs) cause the relocalization of hLigl, PCNA and replication protein-A (RPA), leading to the dispersal of replication factories and to the formation of DNA repair foci. Reorganization of the subnuclear compartments was accompanied by a transient phosphorylation of the p32 subunit of RPA and by a transient dephosphorylation of Ser66 on hLigl. ${ }^{8}$ Taken together, these data indicate that during cell cycle and DNA repair the activity of $\mathrm{hLigl}$ is strictly regulated by phosphorylation/dephosphorylation.

To investigate if the phosphorylation status of human DNA ligase I could be modulated also during the apoptotic cell death, exponentially growing HL60 cells were incubated for increasing times with $68 \mu \mathrm{M}$ etoposide, a topoisomerase II inhibitor which affects DNA replication and activates the apoptotic pathway. The fate of hLigl was followed by two mAbs characterized in our laboratory, the $1 \mathrm{~A} 4$ clone that is specific for the hLigl phosphorylated on Ser66, and the 2B1 clone, which recognizes the antigen irrespectively of its phosphorylation status. Western blot analysis with 2B1 revealed in untreated cells the expected band of $125 \mathrm{kDa}$ corresponding to $\mathrm{hLigl}$ (Figure 1A, lane 1). Etoposide treatment induced a progressive increase in the electrophoretic mobility of the protein, as revealed by the shift in the apparent molecular weight of the enzyme (lanes $2-5$, corresponding to cells treated with $68 \mu \mathrm{M}$ etoposide for $1,2,4$ and $6 \mathrm{~h}$, respectively), thus indicating that prolonged etoposide treatment caused hLigl dephosphorylation. This evidence was confirmed by the $1 \mathrm{~A} 4 \mathrm{mAb}$ directed to hLigl phosphorylated on Ser66. Indeed, a sudden decrease in the level of the phosphorylated protein was visible in cells treated with etoposide for 4 and $6 \mathrm{~h}$ (Figure 1B, lanes 4 and 5). This finding is in agreement with our previous observation on HeLa cells ${ }^{8}$ and suggests that dephosphorylation of hLigl is a general feature of etoposide-treatment. Since we have previously shown that Ser66 is part of a consensus site for Casein Kinase II (CKII; 7), we investigated if hLigl from apoptotic cells could still be a substrate for this kinase. The immunopurification of hLigl from extracts of cells harvested after a $6 \mathrm{~h}$-treatment and the further phosphorylation with CKII, revealed that hLigl was still a substrate for CKII (Figure 1B, lane 6). The analysis of the level of the catalytic $(\alpha)$ and regulatory $(\beta)$ subunit of CKII in extracts from HL60 cells treated with etoposide for 1, 2, 4 and $6 \mathrm{~h}$, showed that CKIl $\alpha$ and $\beta$ were present at comparable levels in untreated and etoposide-treated cells (Figure 1C), thus suggesting that dephosphorylation of hLigl depends on the activation of a phosphatase activity and not on the kinase inactivation. The immunofluorescence analysis of hLigl distribution in apoptotic HL60 cells showed that dephosphorylated hLigl colocalized with the apoptotic bodies (Figure 1D, arrowheads), thus supporting the notion that dephosphorylation increases the affinity of $\mathrm{hLigl}$ for chromatin structures. ${ }^{7}$

To better characterize the cells in which hLigl is dephosphorylated, we monitored a series of apoptotic parameters, including chromatin condensation, DNA fragmentation and PARP-1 proteolysis. In cells showing the complete dephosphorylation of hLigl (i.e. after a 4 and $6 \mathrm{~h}$ treatment with $68 \mu \mathrm{M}$ etoposide), we found respectively about 67 and $88 \%$ of cells with apoptotic morphology, as revealed by Hoechst staining (Figure 1E). Furthermore, we observed in these samples a massive DNA laddering (Figure 1F) and PARP-1 cleavage (Figure 1G). Altogether, these data indicate that complete dephosphorylation of hLigl occurs in cells in the execution phase of etoposide-induced apoptosis. No evidence for this event was obtained in HeLa cells driven to apoptosis by long-term culture conditions.

Protein dephosphorylation has been a rather neglected aspect of cell death and the timing of dephosphorylation events during apoptosis is still controversial. In line with our results on $\mathrm{hLigl}$ in etoposide-treated cells, a change in the degree of phosphorylation of the HMG A1a protein has been shown in advanced apoptotic cells $;{ }^{9}$ on the contrary, histone $\mathrm{H} 1$ subtypes $^{10}$ and Tau protein ${ }^{11}$ undergo dephosphorylation early after apoptosis induction by different stimuli, including topoisomerase inhibitors. It has been reported that during apoptosis dephosphorylated $\mathrm{RB}^{12}$ and $\mathrm{Tau}^{13}$ are cleaved by caspases. Under conditions of PARP1 degradation in etoposide-treated HL60 cells (Figure 1G), we found no evidence of hLigl cleavage. Furthermore, kinase assays revealed that the phosphorylation sites of the protein were conserved (Figure 1B), thus indicating that the structural properties of the enzyme were not affected.

The physiological relevance of protein dephosphorylation during apoptosis is still unknown. As suggested by the observation that the pro-apoptotic protein BAD once dephosphorylated by the serine-threonine phosphatase 
A

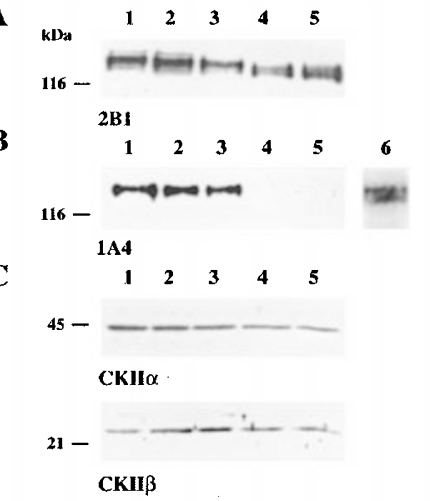

Apoptotic Index

$\mathbf{1 . 3 \%}$

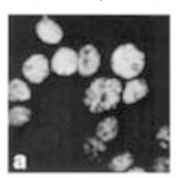

$66.7 \%$

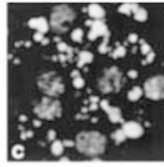

$\mathbf{E}$

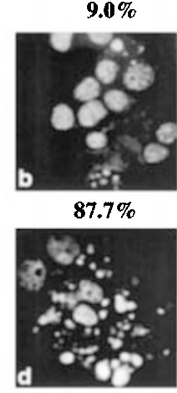

D

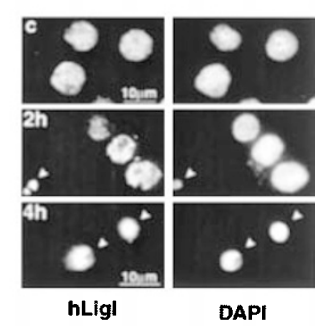

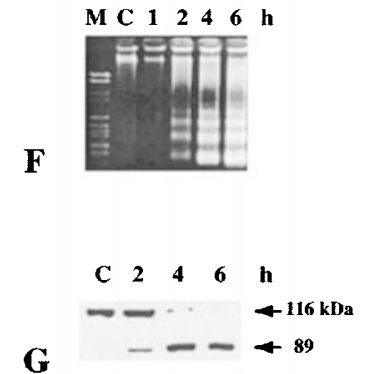

Figure 1 Dephosphorylation of hLigl during etoposide-induced apoptosis Cell extracts, prepared as previously described ${ }^{17}$ from control HL60 cells (lane 1) and from cells incubated with $68 \mu \mathrm{M}$ etoposide for 1, 2, 4, 6h (lanes 2-5 respectively), were analyzed with (A) $2 \mathrm{~B} 1 \mathrm{mAb}$ against hLigl; (B) $1 \mathrm{~A} 4 \mathrm{mAb}$ directed to phosphorylated Ser66 of hLigl; (C) a rabbit antiserum to $\alpha$ or $\beta$ subunit of CKII (kindly provided by Prof. Pinna, University of Padova). hLig was immunoprecipitated as previously reported ${ }^{7}$ from HL60 cells treated for $6 \mathrm{~h}$ with $68 \mu \mathrm{M}$ etoposide and was in vitro phosphorylated by $0.4 \mathrm{mU}$ of CKII in a reaction mixture containing $50 \mu \mathrm{M}$ ATP and $10 \mu \mathrm{Ci}$ of $\gamma^{32} \mathrm{P}$-ATP. Phosphorylated hLigl was visualized by autoradiography (B, lane 6). (D): HL60 cells treated with $68 \mu \mathrm{M}$ etoposide for 2 and $4 \mathrm{~h}$ were dropped on coverslips pretreated with $1 \mathrm{mg} / \mathrm{ml}$ polylysine, fixed with cold methanol and stained with $2 \mathrm{~B} 1 \mathrm{mAb}$ to $\mathrm{hLigl}$ and with a rhodamine-conjugated sheep antimouse IgG antibody as previously described. ${ }^{7}$ Chromatin was stained with $0.2 \mu \mathrm{g} / \mathrm{ml} \mathrm{DAPI}$. Conventional epifluorescence microscopy was performed with a Leitz Orthoplan microscope equipped with a $63 \times$ objective. C: untreated cells. Arrowheads: apoptotic bodies. (E): apoptotic morphology of etoposidetreated HL60 cells stained with $0.1 \mu \mathrm{g} / \mathrm{ml} \mathrm{Hoechst} \mathrm{33258.} \mathrm{a:} \mathrm{untreated} \mathrm{cells;} \mathrm{b-}$ d: cells incubated with $68 \mu \mathrm{M}$ etoposide for 2,4 and $6 \mathrm{~h}$, respectively. The apoptotic index, representing the percentage of apoptotic cell over the tota cell number, was calculated from three independent experiments. (F) DNA laddering was analyzed by agarose gel electrophoresis according to Donzelli et al. ${ }^{17}$ DNA molecular weight marker VI (Boehringer) was used (M). (G): PARP-1 proteolysis was detected as previously described ${ }^{17}$ by Western blotting with the mAb C-2-10 (Alexis). Results of a typical experiment out of three are shown. C: untreated cells

calcineurin, promotes apoptosis, ${ }^{14}$ dephosphorylation could be highly regulated within the apoptotic pathway. From our data, it is tempting to speculate that the activation of the DNA damage-induced apoptotic pathway requires signals to abolish cell cycle progression and to inhibit enzymatic activities involved in the maintenance of chromatin structure and in DNA replication. This has been verified for some proteins involved in chromatin dynamics, such as histone $\mathrm{H} 1$ subtype $^{10}$ and HMG A1a. ${ }^{9}$ Furthermore, a link between cell cycle arrest and apoptosis has been suggested by the reports on $\mathrm{RB}$ dephosphorylation and $\mathrm{G} 1$ arrest during apoptosis. ${ }^{15,16}$ DNA ligase $I$ is phosphorylated in a cell cycle dependent manner and phosphorylation on Ser66 allows to discriminate between a dephosphorylated prereplicative form and a phosphorylated post-replicative form of the enzyme. ${ }^{7}$ We hypothesize that dephosphorylation of hLigl and possibly of other replicative enzymes is a hallmark of the inactivation of the DNA replication machinery typical of DNA damaged-apoptotic cells.

This work was partially supported by a grant from AIRC to AM and from CNR 'Agenzia 2000' (Project CNRC00CA18) to GB.

\section{R Rossi ${ }^{1}$, A Montecucco $^{1}, M$ Donzelli $^{1}, M$ Denegri ${ }^{1}$, G Biamonti ${ }^{1}$ and $A$ I Scovassi ${ }^{*, 1}$ \\ 1 Istituto di Genetica Biochimica ed Evoluzionistica del CNR, Via Abbiategrasso 207, 27100 Pavia, Italy \\ * Corresponding author: Fax: 39-0382-422286; \\ E-mail: scovassi@igbe.pv.cnr.it}

1. Boulikas T (1994) Anticancer Res. 14: 2465-2472

2. Tomkinson AE and Levin DS (1997) BioEssays 19: 893-901

3. Montecucco A et al. (1995) EMBO J. 14: 5379-5386

4. Barnes DE et al. (1992) Cell 69: 495-504

5. Montecucco A et al. (1995) Nucleic Acids Res. 23: 962-966

6. Montecucco A et al. (1998) EMBO J. 17: 3786-3795

7. Rossi $R$ et al. (1999) EMBO J. 18: $5745-5754$

8. Montecucco A et al. (2001) Mol. Biol. Cell 12: 2109-2118

9. Diana F et al. (2001) J. Biol. Chem. 276: 11354-11361

10. Kratzmeier M et al. (2000) J. Biol. Chem. 275: 30478-30486

11. Mills JC et al. (1998) J. Cell Sci. 111: 625-636

12. Fattman CL et al. (1998) Cancer Letters 130: 103-113

13. Canu N et al. (1998) J. Neurosci. 18: 7061-7074

14. Wang H-G et al. (1999) Science 284: $339-343$

15. Dou QP et al. (1995) Proc. Natl. Acad. Sci. USA 92: 9019-9023

16. Chen WD et al. (1997) Oncogene 14: 1243-1248

17. Donzelli M et al. (1999) Oncogene 18: 439-448 